\title{
L'Empire ottoman revisité
}

The Ottoman Empire revisited

\section{Krikor Beledian}

\section{(2) OpenEdition}

Journals

Édition électronique

URL : https://journals.openedition.org/ceb/1520

DOI : $10.4000 /$ ceb. 1520

ISSN : 2261-4184

Éditeur

INALCO

Édition imprimée

Date de publication : 1 décembre 2008

Pagination : 197-211

ISBN : 978-2-85831-173-6

ISSN : 0290-7402

Référence électronique

Krikor Beledian, «L'Empire ottoman revisité », Cahiers balkaniques [En ligne], 36-37 | 2008, mis en ligne le 15 mai 2012, consulté le 06 juillet 2021. URL : http://journals.openedition.org/ceb/1520 ; DOI :

https://doi.org/10.4000/ceb.1520

Ce document a été généré automatiquement le 6 juillet 2021.

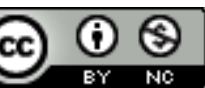

Cahiers balkaniques est mis à disposition selon les termes de la Licence Creative Commons Attribution - Pas d'Utilisation Commerciale 4.0 International. 


\title{
L'Empire ottoman revisité
}

\author{
The Ottoman Empire revisited
}

\section{Krikor Beledian}

1 Cette étude a pour objet l'œuvre monumentale qu'est la monographie Constantinople et son rôle, signée par Hagop Sirouni (Adapazar 1890-Bucarest 1973) parue en quatre volumes, d'environ 2000 pages et restée inachevée ${ }^{1}$. Cette restriction s'impose d'emblée pour des raisons qui seront avancées plus loin.

\section{Repères}

2 Sans entrer dans la discussion pour savoir si la Roumanie fait ou non partie des Balkans, il me semble nécessaire de remarquer que les études sur les diverses communautés arméniennes des Balkans sont rares et, de plus, elles traitent davantage des aspects strictement historiques et presque pas de la dimension littéraire ${ }^{2}$. Cette lacune peut s'étendre à bien d'autres communautés et elle trouve son origine dans la méconnaissance ou la négligence des phénomènes migratoires et de la formation des communautés diasporiques. Considérées souvent comme transitoires, pour ne pas dire volatiles, les communautés diasporiques sont des objets d'études plutôt difficiles, d'autant plus que parfois elles sont éphémères ou en constant changement et échappent à l'analyse. Les ouvrages qui traitent des communautés arméniennes de la Bulgarie, de la Roumanie, de la Hongrie voire de la Grèce, pour ne parler que de ces pays d'Europe orientale, leur consacrent quelques pages assez sommaires ${ }^{3}$ tout en reconnaissant qu'il s'agit de «colonies » ou de «communautés $»^{4}$ fort anciennes, certaines remontant au début du $\mathrm{X}^{\mathrm{e}}$ siècle.

3 Mais si l'historien des communautés diasporiques rencontre des difficultés intrinsèques à sa discipline, l'historien de la littérature peut s'estimer heureux, dans la mesure où il peut s'appuyer sur les œuvres. Cet avantage n'est que théorique, car non seulement les faits littéraires sont souvent négligés et ne sont guère intégrés à l'histoire générale de la littérature arménienne, mais souvent les œuvres, elles-mêmes, ne sont ni cataloguées, ni sériées ou étudiées comme des productions originales dont le contexte, 
en tant que processus littéraire dont elles seraient le fruit, mériterait d'être pris en compte. Comme il m'est arrivé de le signaler pour le cas français ${ }^{5}$, nées à l'étranger et donc en dehors du pays natal ou de la République d'Arménie, les productions diasporiques ont pâti du fait d'être le produit d'une expérience de confrontation avec un environnement nouveau, avec tout ce que cela suppose comme réactions de rejet ou d'attrait.

4 Aussi ne faudrait-il pas m'en tenir rigueur si je fais l'impasse sur le processus littéraire d'une communauté comme celle de Roumanie. Je signalerai que c'est Sirouni lui-même qui, par ses études sur l'histoire et sur les personnalités de sa communauté, nous éclaire le plus. Mais ceci n'est pas l'objet de mon intervention. Je vais me contenter de donner quelques indications indispensables à la compréhension de l'œuvre de cet écrivain aux visages multiples. À la fois, poète, conteur, critique littéraire, historien, intellectuel et publiciste, à lui seul, il a constitué une espèce d'institution vivante. Sa carrière littéraire roumaine se situe entre 1922 et 1973, plus exactement entre la grande vague d'émigration forcée postérieure au génocide de 1915 et la consolidation du communisme en Roumanie sous Caeucescu. Mais c'est certainement l'entre-deuxguerres qui constitue sa période d'activité la plus intense.

Pour l'étude d'une communauté diasporique, l'étude de la presse en langue arménienne est un passage obligé. Le processus littéraire passe par là et on sait que nombre d'écrivains (privés de la possibilité de publier des livres), ne s'expriment que dans la presse périodique, voire la presse quotidienne. Cela est vrai tout particulièrement pour le cas arméno-roumain. Pour une population arménienne arménophone, estimée par Sirouni entre 20.000 et 35.000 personnes, mais disséminées très inégalement dans les diverses provinces roumaines, on trouve une dizaine de publications quotidiennes, hebdomadaires, mensuelles ou périodiques. Trois journaux occupent une place de choix: Nouvelle aube (Nor Archaloys, 1922-1934), Araz (1932-1944) et Nouvelle vie (Nor Guiank), 1950-1980). Il convient d'ajouter les publications en langue roumaine Ararat (1924-1942) et Ani (Revue de culture arménienne, 1925-1938, 1941-1943) Il faut aussi tenir compte des revues plus éphémères comme Massis (Mont Ararat, 1920-1922), Yerguir (Pays, 1924-1928), Navasard (Nouvel an, 1923-26) de Sirouni, Ari (Vaillant, 1924-1928, puis 1934-35), Herg (Sillon, 1937-38) et des almanachs tels que Araz (1933-34), Almanach de l'émigré (1939-41), etc.

6 En général, c'est à travers des groupes plus ou moins hétérogènes que s'élabore une littérature d'exil. Or, dans un article du mois de février 1924, au moment où de nombreux écrivains venant de Turquie sont de passage à Bucarest, pour continuer leur route vers l'Europe occidentale ou pour partir vers le Moyen-Orient ${ }^{6}$, Sirouni déclare :

«Il serait audacieux de dire qu'il existe une vie littéraire en Roumanie. Plus que d'autres colonies la nôtre présentait les conditions favorables à un_travail intellectuel. Une densité élevée d'Arméniens, une vie paisible, un certain nombre d'intellectuels : tout cela aurait dû suffire pour entamer un travail intense. Malheureusement le résultat n'est pas celui que l'on attendait $»^{7}$.

7 Malgré la précocité de ce constat, on peut dire que Sirouni n'est pas très optimiste quant au développement d'une littérature d'exil, du moins en Roumanie ${ }^{8}$. Naturellement il fait abstraction de l'œuvre qu'il est en train d'élaborer lui-même. 


\section{Origines}

Il convient de faire un pas en arrière et de revenir à la biographie de Sirouni, alias Djololian. Né à Constantinople (ou Bolis, comme disent les Arméniens), dans la capitale de l'Empire ottoman, en 1890, il fait ses études au Collège central. Son adolescence correspond à la fin du règne d'Abdul Hamid II et aux remous révolutionnaires. A-t-il déjà adhéré aux idées nouvelles qui agitent les cercles clandestins de la capitale ? Objet de suspicion de la police, Sirouni connaît l'enfer des prisons ottomanes, mais il est libéré après quelques mois9. À cette époque il fréquente les milieux littéraires de la capitale et se lie d'amitié avec le jeune poète Missak Medzarents (1886-1908), le poète Vahram Thatoul (1887-1943) l'écrivain Kegham Barseghian (1883-1915) et le journaliste Chavarch Missakian (1884-1957).

9 Sa carrière littéraire commence par la publication de Crépuscule ${ }^{10}$ un premier recueil de poèmes écrits dans l'esthétique symboliste chère à cette génération; ses premiers poèmes remontent aux années 1905-1906. D'autres plaquettes suivront ${ }^{11}$, jusqu'au recueil Vers le temple du miracle en 1914 où Sirouni célèbre la Beauté et adhère à l'esthétique « néopaïenne » de son ami Daniel Varoujan (1884-1915). Avec ce dernier, il publie l'Almanach Navasard («Nouvel an », Constantinople, 1914), tout en restant à l'écart du mouvement littéraire initié par la revue d'avant-garde Méhian où certains de ses amis se retrouvent. Cette veine poétique assez abondante qui penche parfois vers l'utilisation des thèmes folkloriques ${ }^{12}$ reprendra après l'Armistice par la publication de nombreux poèmes jamais repris en volume.

Entre-temps les événements politiques se précipitent. L'entrée en guerre de l'Empire ottoman aux côtés des puissances de l'axe met fin à l'effervescence littéraire des Arméniens de l'Empire. Le journal Azatamart (Combat) auquel collabore Sirouni est fermé par les autorités Jeunes-Turques. La majorité des intellectuels en vue sont arrêtés. Sirouni est l'un des rares à pouvoir entrer en clandestinité où il restera jusqu'à la fin de la guerre et n'en sortira qu'au mois de décembre 1918. C'est ainsi qu'il échappe à l'anéantissement programmé, «à la Catastrophe ${ }^{13}$ qui laissera des traces indélébiles sur son œuvre comme sur sa pensée.

11 Durant la courte période 1919-1922, au cours de laquelle l'intelligentsia survivante croit pouvoir reprendre, retrouver les conditions d'une vie intellectuelle normale dans l'Empire ottoman, Sirouni reprend sa collaboration à la presse arménienne, publie articles, poèmes et s'intéresse au théâtre. Mais dès 1920 on le trouve à Salonique, puis en Bulgarie, finalement à Bucarest en Roumanie où, au mois d'octobre 1923, il lance la revue Navasard (Nouvel an), revue de littérature et d'art. Les écrivains réunis autour de cette revue sont ceux qui viennent de fuir la Turquie et sont plus ou moins disséminés en Europe et dans les Balkans ${ }^{14}$. Sirouni et ses amis (le père Bodourian et l'intellectuel H. Athanassian) fondateurs de la revue semblent partager les préoccupations esthétiques de l'Almanach Navasard de 1914 : la nostalgie du passé est déjà en œuvre ${ }^{15}$ et elle est d'autant plus prégnante que tout retour est impossible. Pour pouvoir gérer la rupture et intégrer la perte, les exilés se sentent contraints à rétablir les chaînes de la transmission.

12 Car la situation des Arméniens a profondément changé : le désastre de 1915, la chute de la première République arménienne (1918-1920), la débâcle de 1922, le traité de Lausanne et la dispersion rendent, plus que jamais, la littérature et l'art indispensables. 
Voici ce que nous déclare Sirouni dans l'éditorial du premier numéro qu'il intitule «Pour l'indépendance des âmes »:

"Le peuple arménien vit l'une des périodes les plus tragiques de son histoire - des jours de déception et de désespoir... Mais qui dit que ce peuple va mourir. Dans son histoire séculaire, il a eu des périodes semblables de catastrophe et de souffrance. Mais à chaque fois il est né des cendres comme un phénix... Non, ce peuple arménien ne mourra pas... C'est cette foi qui nous lance dans l'arène. Le peuple arménien n'est pas mort parce qu'il a aimé l'art. Il l'a aimé d'un amour et d'une ferveur sans limites... Et l'art l'a sauvé, car l'art l'a doté d'une force pour résister à la souffrance... Qui sommes-nous? Quelques jeunes gens qui croient que sans la beauté un peuple ne pourra pas vivre, que les survivants du massacre et de la famine se feront écrasés et seront brisés si les mères oublient leur berceuse et le paysan son chant, que le peuple arménien ne pourra durer un jour de plus s'il oublie le conte que lui a raconté l'Ararat".

Dans un deuxième article intitulé "Au nom de la fraternité des hommes », Sirouni revient sur l'importance de l'art après les quatre ans de guerre :

"L'art est l'unique force qui tend un pont inébranlable entre les siècles et les races, démolit les murailles que les hommes ont construites pour leurs passions terrestres.

C'est la main magique de l'artiste qui brise toutes les frontières...

Les religions et les Dieux meurent, les rois et les sages descendent sous la terre, les palais se brisent, les vérités inventées par les hommes sont réduites en cendre... la Beauté seule est grande et éternelle... car son temple est le seul que les hommes n'ont pu profaner ».

L'auteur de ces lignes reste parfaitement convaincu que l'art et la littérature sont encore possibles après le désastre et il s'attache à les promouvoir dans les limites qui sont les siennes. Aussi bien sur le plan théorique que sur le plan pratique d'écriture, Sirouni demeure un exilé qui regarde en arrière. Tout d'abord, en matière d'écriture, Sirouni abandonne progressivement la poésie pour la prose et, entre 1922 et 1925, il se met à publier des contes symboliques.

\section{Contes et oubli}

15 La série porte le titre général de « Les histoires du Derviche » et se présente comme des récits entendus de la bouche d'un Derviche, il y a plus de trente ans, pendant l'enfance du narrateur ${ }^{16}$. Le texte évoque les aventures du Derviche, ses déboires, ses déceptions, ses expériences vécues d'où sont tirées des vérités générales. Dans « Ce que la Caravane a enduré $»^{17}$, le Derviche enseigne que Dieu, Allah, ne réside pas dans la mosquée, mais dans le cœur des hommes qui lui ont donné leur propre image, etc. Ces récits écrits comme des histoires rapportées reprennent le genre du conte oriental plus ou moins enchaîné. Si le monde contemporain est absent de ces histoires, il n'en reste pas moins à l'arrière-fond et surgit dans la série "Les histoires de la vieille femme $»^{18}$ qui sont des récits également rapportés, mais de la bouche d'une vieille femme qui rendait visite au narrateur pendant sa clandestinité. Ce dernier s'abstient de raconter longuement ses années de clandestin recherché et traqué par la police tout en l'évoquant d'une manière sommaire. La vieille femme est une inconnue qui rend visite aux clandestins réfugiés dans des familles accueillantes et son rôle est justement de leur tenir compagnie, de les aider à sortir de leur solitude, de leur abattement, en leur racontant des histoires. La réalité hostile et dangereuse du monde extérieur est ainsi abolie, effacée, oubliée, alors que l'imagination prend son élan, créant un «monde nouveau à la place des ruines anciennes ». 
16 Il est évident que ces contes jettent dans l'abîme le propre travail de Sirouni. Ils permettent à l'exilé, comme ils le faisaient au clandestin d'antan, de s'abstraire d'un environnement difficile, de se créer un monde de fiction qui protège le rescapé, de se construire une mémoire et de revivre les moments fastes de sa vie antérieure. La conclusion du premier conte est très significative. Le narrateur s'adressant à ses lecteurs, aux «jeunes gens", déclare : "Voyez comme je souffre maintenant pour que les tentations du monde ne me fassent pas oublier les paroles douces et amères que m'a contées la vieille femme et pour que je puisse aménager l'édifice que je me suis mis à construire dans mon âme $»^{19}$. Le récit est un écran protecteur, voire un abri, un refuge où l'exilé tente de se loger, entouré d'un monde désertifié et vide.

17 L'exilé est un être déchiré, on le sait, et il n'est pas évident que la recherche d'un oubli salvateur soit le moyen le plus approprié d'échapper aux effets pervers de la catastrophe. En effet, Sirouni ne peut oublier les lieux, les visages qu'il a connus, les événements qu'il a vécus. Si les contes tentent d'élever un mur protecteur autour du sujet, ce dernier ne cesse d'être rattrapé par son passé. Aussi dans chaque numéro de sa revue Sirouni se fait un devoir d'évoquer les écrivains " disparus ", les " acteurs » et autres intellectuels, ses compagnons morts en déportation. Il consulte ses archives et publie des textes inédits, des photographies, des documents, justement pour que ces hommes ne soient pas complètement anéantis. Bien plus, il commence à rédiger ses souvenirs, qui paraissent à Boston ${ }^{20}$. Intitulé La Génération d'Azatamart, ce texte traite des principaux événements qui ont entouré la naissance du journal, des collaborateurs, des problèmes que la rédaction a dû affronter jusqu'à l'interdiction finale, au mois d'avril 1915, de l'arrestation des écrivains jusqu'à la fuite et l'entrée en clandestinité du narrateur Sirouni. Dans la préface du texte Sirouni met le doigt sur cette "épidémie ", cette prolifération d'une littérature de "souvenirs ": "Nombreux sont ceux qui évoquent le passé : la préoccupation de tous est la même : ne pas laisser le passé mourir [...]. La génération qui survit [...] a le devoir de sauver ce qui peut l'être encore. Encore quelques années, encore quelques morts parmi les anciens et le passé va disparaître au fond du brouillard [...]». Évoquant son propre projet, Sirouni précise qu'il n'a pas la prétention d'écrire des souvenirs, qu'il n'en a guère les moyens, ni l'audace, ni l'âge. Il veut, dit-il, «ne donner que l'image, autant que le permettent [sa] mémoire et [son] jugement, d'une époque où Azatamart vécut et tracer les traits de la génération qui l'a créée [...] ». «Je serais heureux, dit-il, si je pouvais donner les traits authentiques de cette vie et quelques notes présentant une certaine valeur, aux véritables historiens ${ }^{21}$. Comme d'autres, mais avec une conscience plus aigüe de la perte, Sirouni entame son récit. De fait, c'est là, dès la fin des années vingt, que le mémorialiste fait ses premières armes, avec l'intention affichée de «sauver ce qui peut l'être encore [...] car demain ce sera trop tard».

18 Avant de franchir définitivement le pas, Sirouni publie une pièce de théâtre dans la revue Hayrénik (Patrie) de Boston, en 1927. La tentation théâtrale remonte aux années 1912-1914, où Sirouni avait déjà rédigé deux œuvres, jamais portées sur scène ${ }^{22}$. Dans un entretien qu'il a accordé à la revue Chanth, en 1919, il nous apprend qu'en "ce moment » le théâtre l'intéresse et qu'il a déjà écrit déjà trois pièces pendant sa clandestinité23. L'obsession théâtrale n'est pas propre à notre auteur, il la partage avec les écrivains de sa génération, tels que K. Zarian (1886-1969) et H. Oshagan (1883-1948) qui, à la même époque, tentent de créer un « répertoire national ». La pièce de Sirouni Et Ceux qui vivent s'inscrit dans ce contexte de « redressement $»^{24}$. En trois actes, l'action 
a lieu à Constantinople entre 1915 et 1918, dans une grande famille bourgeoise arménienne. La tragédie oppose une poignée de victimes des persécutions turques aux richissimes commerçants de la capitale, prêts à tout, y compris la délation, pour sauver leur fortune et leur situation. Ce tableau des aliénations dénonce la collaboration de certains Arméniens au processus d'anéantissement ${ }^{25}$. L'ouvrage oscille entre la satire, le sarcasme et les larmes, Sirouni ne parvenant pas toujours à éviter le mélodrame. Sans être un témoignage, l'ouvrage contient des indications rares et précieuses sur une période que l'on connaît assez mal: la vie des Arméniens dans la capitale ottomane dans les années 1915-1918.

\section{Monument}

19 Dans les années trente Sirouni publie fort peu de textes à caractère littéraire ${ }^{26}$. Avec son collègue et ami roumain N. Iorga, il lance la revue Ani en roumain, fait des traductions de la poésie roumaine (Mihail Eminesku) ou écrit des articles sur divers aspects de la culture arménienne ${ }^{27}$. C'est probablement à cette époque que son écriture commence à proliférer dans tous les sens et que des études arménologiques sont entreprises, alors que le mémorialiste ne cesse de publier ses souvenirs dans la presse arménienne de France, des États-Unis ou de Turquie. Son ouvrage Tels qu'ils étaient: Daniel Varoujan (Bucarest 1940) est une mine d'informations sur le poète ami, et lui permet de jeter un regard renouvelé sur cette période de sa jeunesse.

20 En fait, Constantinople est pour lui une fascination, une obsession ${ }^{28}$. Dès 1936, Sirouni intervient dans la polémique lancée par le journal Hayrénik de Boston sur le rôle jugé néfaste de l'ex-capitale de l'Empire ottoman dans l'histoire des Arméniens. Il consacre une dizaine d'articles à sa défense ${ }^{29}$. C'est en réalité l'amorce du futur ouvrage. Mais avant l'élaboration de ce dernier, Sirouni, resté en Roumanie, est arrêté en 1944 par les agents de l'Armée Rouge. Malgré les nombreux articles qu'il avait publiés contre le nazisme et l'idéologie raciale, taxé d'antisoviétisme il est emmené en URSS où il est condamné à dix ans de camp de concentration. Il a la chance d'être envoyé d'abord en RSS d'Arménie où il reste jusqu'en 1947 avant de purger sa peine dans un camp sibérien ; il ne rentre en Roumanie qu'en 1955. Entre-temps, sa bibliothèque, ses archives et ses manuscrits ont disparu. À son retour, Sirouni semble avoir renoncé définitivement à la littérature. Mais le projet de son livre sur Constantinople s'est précisé. En effet, pendant son séjour à Erevan en 1947, « dans des conditions peu agréables », nous dit-il, «Bolis le hante» et il se met à esquisser un ouvrage dont les premières livraisons paraissent d'abord en Arménie, dans la revue du Catholicossat d'Etchmiadzine, dont le titulaire n'est autre qu'un de ses disciples, Vazken I ${ }^{\text {er }}$ Baldjian (1955-1994). Bénéficiant de l'appui total de la plus haute autorité spirituelle de l'Église arménienne, Sirouni s'adonne à cette tâche immense qu'il remet sans cesse sur le métier, reprend les chapitres publiés, les enrichit, les développe, sans pour autant venir à bout de l'ouvrage, laissant le cinquième volume inachevé.

21 Dans la préface "Entretien avec le lecteur ", ${ }^{30}$ Sirouni tente de définir son but et de lever les ambiguïtés de son projet qui est de « raconter Constantinople ». Il revient sur le fait qu'il a "ruminé ce sujet depuis longtemps ». Dans ces retours en arrière, ces réminiscences se manifestent aussi bien le souvenir que la souffrance de la nostalgie, voire la conscience "que nous n'avions pas connu Constantinople lorsque nous y sommes nés, nous ne l'avions pas connu dans ses jours anciens comme dans son 
présent ». Il raconte Constantinople de dehors, « en terre roumaine où les événements nous ont poussés il y a quarante ans ». Dans cette distance temporelle et spatiale, il trouve cependant un certain avantage pour ne pas dire un réconfort: «Quarante années constituent un temps suffisant pour que l'on oublie beaucoup de choses et qu'on se rappelle de beaucoup d'autres. Qu'on oublie les choses insignifiantes et qu'on se souvienne de celles qu'on n'était pas parvenu à voir quand on en était proche ». Aussi, poursuit-il, "nous commençons à raconter Constantinople à un moment où depuis longtemps nous en sommes éloignés, c'est-à-dire au moment où nous pouvons désormais voir avec discernement et juger avec sang-froid, plus que nous n'aurions pu le faire si nous étions en son sein, participant à ses émotions en témoin oculaire de ses tentations".

Le ton affectueux avec lequel Sirouni monologue à propos de Constantinople, à propos de son Bolis, de sa ville natale et de ce qu'il projette d'écrire souligne la relation nostalgique qu'il entretient avec la capitale de l'Empire ottoman. À ce ton affectueux vient s'ajouter l'humilité, car Sirouni se présente non pas tant comme un historien, mais comme un «humble intellectuel arménien " qui "considère comme un devoir sacré pour lui de sauver quelque chose de Bolis, de ces cinq siècles... $»^{31}$, si riches et si importants pour la culture arménienne. Ce retour symbolique devient propice à l'évocation rapide des souvenirs de jeunesse (les années de lycée, la répression hamidienne, l'enthousiasme de la proclamation de la Constitution) que vient conclure le deuil de 1915. Sirouni se propose de raconter cette époque " qui mit fin à cinq siècles d'espoir et qui terrassa Bolis dans ses efforts et son rôle des jours de gloire ». Comme, dit-il, le peuple arménien n'a pas de mur de lamentation, «ce livre sera un monument pour cette génération » annihilée $e^{32}$. Il est aisé de constater que l'auteur érige un livremémorial, une espèce de cénotaphe pour ceux qui ont disparu à jamais.

Ayant mis son cœur à nu au seuil de son livre et dévoilant par là les éléments propres à un «travail de deuil», à vrai dire impossible, Sirouni ne pense pas moins aux contraintes d'un ouvrage à caractère historique. Car raconter Bolis c'est d'abord reconstituer l'histoire de la présence des Arméniens dans la capitale de l'Empire ottoman. La quête des documents, la recherche des sources, le parcours des travaux déjà effectués sont des étapes indispensables pour cette monographie historique. Sirouni mettra à contribution aussi bien les informations contenues chez les voyageurs européens que des renseignements fournis par les sources ottomanes, et par les écrivains arméniens du XVII ${ }^{e}$ et XVIII ${ }^{e}$ siècles. Il consultera les ouvrages de tous ses prédécesseurs pour finir par constater qu'aucun n'envisage Constantinople comme une totalité à saisir, que ce sont là des approches partielles, qu'il faut parvenir à une saisie synthétique de cinq siècles d'histoire, de culture, de littérature et d'art. Il reproche à ses prédécesseurs d'avoir oublié le contexte, notamment la politique extérieure et intérieure des Sultans, comme il reproche aux historiens turcs leur manque d'objectivité33.

24 Le projet de Sirouni est empreint d'une visée encyclopédique, car il sait que quiconque raconte Constantinople raconte aussi cinq siècles d'histoire des Arméniens et des Ottomans. C'est bien tout l'Empire qu'il s'agit de visiter. Mais pour le moment, au début de son livre, il prend pour ligne directrice et comme objectif de recherche le patriarcat arménien fondé en 1461. C'est autour de ce pivot que gravitent les recherches de l'auteur qui élargit sans cesse son champ d'investigation. Les deux premiers volumes (XV ${ }^{\mathrm{e}}$-XVIII ${ }^{\mathrm{e}}$ siècles) sont certainement les parties les plus travaillées. Sirouni n'est pas 
Michelet, même s'il est emporté par un même élan romantique. Il n'a ni la force intellectuelle, ni l'appui institutionnel de ce dernier. Cela peut expliquer en partie la faiblesse relative des volumes III et IV dont certains passages sont constitués de longues citations, certes précieuses, mais présentées sans distance critique. Le volume IV fait davantage songer à un patchwork qu'à un ouvrage rédigé d'une manière suivie. Il traite du milieu du XIX ${ }^{e}$ siècle : de la formation de la langue moderne, du théâtre, de la littérature, de la fondation des écoles, mais également des questions politiques placées dans le contexte général de l'histoire de l'Empire ottoman. Il faut bien noter que si pour l'histoire de la culture arménienne il dispose de sources assez abondantes, en ce qui concerne l'histoire des relations arméno-turques, les données dont il dispose et qu'il utilise sont nettement insuffisantes, n'ayant accès ni aux archives ottomanes, ni à celles des chancelleries occidentales.

En fait, si l'historien, le sociologue des mentalités, le spécialiste de la littérature peuvent être déçus, le lecteur qui veut pénétrer dans les arcanes de la ville mythique y trouve son compte ${ }^{34}$. La grande Histoire y côtoie l'anecdote, l'analyse suggestive, les citations. Sirouni ne fait parfois que souligner ${ }^{35}$. Vers la fin du quatrième volume, au fur et à mesure qu'approche le moment crucial, la date fatidique des massacres de 1895, où en tant que narrateur il devient contemporain des événements qu'il rapporte, on sent surgir une crise. L'auteur est parfaitement conscient des difficultés théoriques et historiographiques que soulève l'apparition du témoin dans l'historien. À la fin de sa préface, il remarque, presque à regret: "Pour les hommes qui relatent les faits du passé il n'y a pas de moment plus pénible que celui où ils en arrivent à leur époque. Et, à ce moment-là, une tentation se dresse soudain devant eux. C'est vrai qu'ils sont plus informés des événements qu'ils racontent que ceux qui, plus tard, vont se mettre en quête de leur proie dans les collections de journaux, ils n'ont pas la difficulté de séparer le faux du vrai, mais ils ont la difficulté de rester impartial pendant l'analyse des événements et d'oublier un instant les opinions qui ont été les leurs, les sympathies et les antipathies envers les événements et les personnages dont ils ont été les contemporains. Le cas devient encore plus atroce quand celui qui rédige un écrit à propos des événements du passé a eu lui-même un contact personnel avec tout ce qu'il relate. [...] Aussi impartial qu'on veuille bien être, il est difficile de rester sincère pour juger surtout les personnalités avec lesquelles on a collaboré jadis... C'est un labeur héroïque que celui d'être son propre juge ». Lieu commun de l'homme à la fois acteur et historien de ses gestes? Sirouni nous fait pressentir la difficulté qui guette la fin de son ouvrage.

Certes, dit-il, il n'écrit pas de "souvenirs ». Celui qui écrit des mémoires n'est pas " entravé » ${ }^{36}$ de préoccupations de cet ordre. Mais «le rôle et le devoir de celui qui fait des recherches sur des sujets historiques sont différents de ceux du mémorialiste, même si, en abordant les événements contemporains, il bénéficie de ses connaissances et de ses expériences personnelles [...]. Il faut que les tombes nous laissent tranquilles $»^{37}$.

27 Cette dernière phrase qui fonde l'objectivité historiographique sur des tombes fermées jette une lumière toute crue sur l'inachèvement du monument de Sirouni.

28 Il faut d'abord écarter l'hypothèse selon laquelle l'âge ne permettait plus à l'auteur de terminer son ouvrage. Il suffit de regarder son abondante bibliographie. Après les premiers volumes de Constantinople et de son rôle, Sirouni a écrit des textes majeurs qui sont plus ou moins liés à cette période cruciale. D'abord La Catastrophe et son histoire, 
ensuite Avec Komitas ${ }^{38}$, ce dernier texte est en soi un ouvrage nouveau doté d'une préface dont les préoccupations rejoignent celles exprimées dans Constantinople et son rôle. Il s'agit toujours de sauver, mais dans le cas présent, un personnage clef de la période 1909-1915 et une figure symbolique. Sirouni livre des «notes », dit-il, mais qui sont très longuement élaborées alors qu'en même temps il laisse son monument inachevé ${ }^{39}$.

Dans ce cinquième volume, il aurait dû raconter le Constantinople du 11 avril ${ }^{40}$ qu'évoque déjà $\mathrm{La}$ Catastrophe et son histoire et qu'il avait abordé dans la Génération d'Azatamart (1924). Or, ce dernier texte, comme le Komitas, s'apparente davantage à un témoignage personnel qu'à un récit historiographique. Dans ces deux textes, c'est le regard du témoin qui prime plutôt que l'analyse de l'historien. Le ton est davantage celui de la narration " autobiographique » que celui du récit « objectif ». Tout se passe comme si, pour parvenir au bout de son entreprise, Sirouni - acteur et narrateur - se trouvait devant une aporie insoluble : le fait de devoir achever un ouvrage à caractère historique par un témoignage personnel ; adopter le style serein de l'historien ou bien laisser libre cours aux affects du témoin. L'histoire n'était pas achevée, le témoin n'avait pas encore tout dit, le rescapé qui avait miraculeusement survécu en clandestinité demeurait tapi derrière les mots. Les tombes n'étaient pas fermées. Visiblement elles ne laissaient guère le narrateur tranquille. Le deuil n'était plus possible. Les documents, les sources, les preuves se trouvaient refoulés par un discours qui n'avait sa justification que dans l'expérience personnelle du narrateur-auteur. L'échec de Sirouni - si échec il y a - réside dans l'impossibilité à concilier Histoire et mémoire, à porter le témoignage comme le comble de l'histoire. Sans doute sa propre conception de l'écriture historique était-elle trop traditionnelle et empêchait l'élaboration d'une approche nouvelle et non conformiste.

Le monument de Sirouni présente un Empire ottoman plein d'espoir à partir de 1839 qui finit par l'anéantissement non seulement des espoirs, mais plus encore par la liquidation des Arméniens. Pourra-t-on dire que le résultat est un ouvrage historique ? Les mises en garde, les précautions oratoires de l'auteur ne trompent pas. Constantinople et son rôle est un livre inclassable, un de ces livres où la passion de la connaissance encyclopédique finit par englober l'auteur comme pour l'étouffer. Si la fiction en est bannie, les efforts de reconstitution du passé, les interventions personnelles de l'auteur, puis le risque, certes écarté du témoignage, donnent à l'ouvrage une indéniable dimension littéraire. Sirouni visite l'Empire ottoman et sa capitale, comme jadis Yérémia Tchélébi, son illustre prédécesseur l'avait fait dans son Histoire de Stambol $^{41}$. Il fait un état des lieux, parcourt l'espace et le temps, pour revenir au lieu de son exil. Envisagé sous cet angle, le monument érigé par Sirouni est autre chose qu'un livre de plus sur les Arméniens de l'Empire ottoman. 


\section{NOTES}

1. Le titre arménien est: Bolis yew ir tere, Volume I Beyrouth, 1985 ; volume II, Beyrouth, 1970 ; Volume III, Antélias, 1987 ; Volume IV, Antélias, 1988.

2. Sur l'histoire des Arméniens en Roumanie, il existe un ouvrage fort bien documenté, cf. S. Kolandjian, La communauté arménienne de Sutchava, Erévan 2004 (en arménien). Voir également N. Iorga, Les Arméniens de Roumanie, Bucarest, 1929.

3. Il existe deux ouvrages d'intérêt inégal: A. Abhrahamian, Ébauche d'une histoire des colonies arméniennes (2 volumes, Erevan, 1867) et H. Adjarian, Histoire de l'émigration arménienne, Erevan, 2002.

4. Les termes arméniens "gaghouth ", "gaghthavayr " ou " hamaynk» apparaissent bien avant la grande dispersion de 1915, donc avant l'apparition du terme « diaspora (spurk) » et les auteurs ne sont pas toujours sensibles à l'évolution du vocabulaire correspondant souvent à des changements profonds dans les mentalités.

5. Cf. Krikor Beledian, Cinquante ans de littérature arménienne en France, du Même à l'Autre, CNRS Éditions, Paris, 2001.

6. En l'occurrence le satiriste Y. Odian, l'écrivain M. Chamdandjian, etc.

7. Navasard, Bucarest, $\mathrm{n}^{\circ} 8$, février 1924, p. 244.

8. Observateur attentif de ce qui se passe en Europe, Sirouni en arrive à des conclusions différentes pour ce qui est des nouveaux écrivains arméniens de France, cf. Beledian, Cinquante ans de littérature arménienne en France, op. cit. pp. 74-75.

9. Cet épisode de sa vie est relaté dans une série d'articles parus dans Azatamart, Constantinople, 1914.

10. Metenchagh, 1908.

11. Requiem 1908, Après la nuit 1908.

12. À cette époque Sirouni recueille des poèmes populaires de mariage, cf. Sourhantag (Messager), Constantinople, octobre-novembre 1907 et plus tard il portera un intérêt croissant pour la poésie des « achough » (troubadours), l'épopée orale David de Sassoun et la poésie médiévale arménienne.

13. «Catastrophe" ou "désastre " (Aghed), que Sirouni emploie à l'instar de nombre de ses compagnons, sont les noms "propres» du génocide de 1915. Pour le terme ainsi que la problématique générale d'une écriture de la catastrophe, cf. «L'expérience de la catastrophe dans la littérature arménienne", in Revue d'Histoire arménienne contemporaine, Paris 1995, et "L'expérience des limites du langage », in Parler des camps, penser les génocides, Textes réunis par C. Coquio Albin Michel, 1999. Je renvoie également à l'article de M. Nichanian «L'Europe et l'Empire. Origine de la catastrophe ", in L'Ange exterminateur, édité par J. Gilibert et P.Wilgowicz, Bruxelles 1993.

14. Sur cette littérature de la diaspora, voir Beledian, Cinquante ans de littérature arménienne en France, op. cit.

15. Le premier numéro comprend un texte «Une décennie » qui rappelle l'Almanach Navasard publié jadis avec le poète Varoujan, op.cit., p. 3.

16. Cf «Les histoires du Derviche", Préface, in Nor Charjoum (Nouveau mouvement) $\mathrm{n}^{\circ} 18$, Le Caire, 1923.

17. Cf. Hayrénik, mensuel, Boston, avril 1924.

18. Cf. Navasard, Bucarest, $1924, n^{\circ} 9$, pp. 232-236 et $n^{\circ} 10$, pp. 273-277.

19. Navasard, $\mathrm{n}^{\circ} 9$, p. 201.

20. Hayrenik (la patrie), à partir du numéro de janvier 1924.

21. Ibid., p. 70. 
22. De ces deux pièces que leur auteur croyait perdues après les perquisitions de ses archives par les communistes roumains, en 1944, une seule a surgi depuis, intitulé Le nid d'aigle. Il s'agit d'un " essai dramatique en un seul acte ", écrit en 1912, à Constantinople (cf. Archives Sirouni, nº 39 du Catalogue du Musée d'art et de littérature d'Erevan, Arménie).

23. Chanth, Constantinople, 1919, mars, $n^{\circ} 20$, p. 237. Ces pièces s'intitulent: Que la lumière soit ; $N^{\prime}$ accorde pas de la lumière et Le Derviche. Ces œuvres n'ont jamais été publiées et sont considérées comme perdues. Le catalogue des œuvres établies par S. Kotcharian les mentionne parmi les œuvres perdues, cf. Banber Hayastani Arkhiwneri, Erévan, 1977, nº 3, p. 202.

24. Voir Beledian, «Le retour de la catastrophe », in L'Histoire trouée, Négation et témoignage, op. cit. 25. Le thème est largement exploité par $\mathrm{H}$. Oshagan dans ses deux pièces Le parrain (1921) et Combat de coqs (non datée), in Trois pièces, San Francisco 1990.

26. Le rythme de sa production est phénoménal: sa signature apparaît dans presque toute la presse de la diaspora arménienne, à Paris, à Boston, en Égypte voire en Turquie dans des domaines aussi variés que l'histoire des Arméniens en Roumanie, les origines du théâtre arménien, les relations turco-roumaines, etc. Je mets à part sa production en roumain, langue que je ne maîtrise pas.

27. Pour des raisons évidentes, nous ne pourrons pas aborder ici les divers aspects de l'œuvre arménologique de Sirouni dont il existe une liste établie sous sa direction par S. Kolandjian, «Bibliographie des travaux arménologiques du Prof. H. Sirouni », in Etchmiadzine, 1971, nº 1.

28. On pourra lire à ce sujet Beledian «Faszination Konstantinopel», in Armenien Wiederendeckung einer alten Kultulandschahft, Catalogue de l'exposition, Museum Bochum, 1995.

29. Ces articles sont parus dans Araz (Araxe, Bucarest), du 22 octobre 1936 au 20 janvier 1937.

30. Volume I, pp. 7-19.

31. Ibid., p. 9.

32. Ibid., p. 11.

33. Ibid., pp. 27-28.

34. Pour une histoire de la littérature une entreprise analogue a été tentée par le grand écrivain et critique Hagop Ochagan (1882-1948) avec le Panorama de la littérature arméno-occidentale (dix volumes), Jérusalem 1945-Antélias 1982.

35. Cela fait penser à l'immense effort de Walter Benjamin dans son projet du Livre des passages, Paris, capitale du XIX ${ }^{e}$ siècle, cf. Cerf, 1997.

36. Pour tout ce passage, cf. pp. 18-19.

37. Ibid., p.19.

38. Ces deux textes sont parus dans Etchmiadzine, le premier en 1965 ( $n^{\circ} 2-4$ et $n^{\circ} 5-7$ ), le second dans plusieurs numéros de la même revue, 1976, nº 1 à $1969, n^{\circ} 5$. Sirouni avait déjà abordé le thème de l'anéantissement de sa génération dans des textes tels que "Le crime d'Avril/ Souvenirs d'un survivant ", in Vem, Paris, 1934, $\mathrm{n}^{\circ} 2$ mars-avril, pp.1-12.

39. Sirouni a écrit également une monographie de Monseigneur Karékin Khatchadourian, patriarche des Arméniens de Turquie (1880-1961), publiée en revue en 1960 et reprise en volume, Éditions Aras, Istanbul, 2003.

40. L'éditeur du volume III note que ce dernier volume, dont on ne dispose que de fragments impropres à une publication, aurait dû s'intituler La catastrophe et son histoire, cf. vol. III, p. XV. Il faut se souvenir qu'à la mort de Sirouni ses archives ont été placées sous séquestre et visiblement emportées puis déposées à la Bibliothèque centrale de Bucarest.

41. Yerémia Tchélébi Keumurdjian (1637-1695) est l'auteur prolifique d'une Histoire de Stambol, d'une Histoire des rois osmanlis, d'une Histoire de l'incendie de Stambol, d'un très précieux Journal (1648-1662), ouvrages tous édités, mais non traduits en une langue occidentale. 


\section{RÉSUMÉS}

L'Empire ottoman dans l'œuvre monumentale d'Hagop Sirouni (Djololian, Turquie 1890-Bucarest 1973), une image qui fascine et déchire. Poète, prosateur, théoricien de la littérature et historien, exilé en Roumanie en 1923, Sirouni édite la revue Nawasart (1924-25) et d'autres publications littéraires en langues arménienne et/ou roumaine. Arrêté en 1944 et condamné à dix ans de camp sibérien, Sirouni retourne à Bucarest au début des années cinquante. Obsédé par la nostalgie d'un pays perdu et hanté par l'épouvante des années 1915-1918, Sirouni entreprend l'évocation de la fin de l'Empire dans des récits et des pièces de théâtre des années vingt et trente. Il fait de l'art une modalité de la survie. Mais cette écriture se mue progressivement en mémoires et en reconstitution historique (Constantinople et son rôle, quatre volumes), comme si le regard tourné vers l'origine se refusait désormais les charmes de la fiction et de l'autobiographie pour s'adonner à un examen apparemment plus distancé, à une appropriation plus critique de la naissance et de la mort de l'Empire. Le récit historique se substitue au récit littéraire. On a parlé souvent d'un renoncement à la littérature, en ce qui concerne ce revirement. Et pourtant, l'Empire ottoman revisité dans ses archives ne cesse pas moins d'être l'objet désiré dont l'image fascine et déchire l'exilé doublement persécuté que fut Sirouni.

This lecture is dedicated to the monumental works of Hagop Sirouni (Djololian, Turkey 1890Bucarest 1973), a poet, novelist, litterary theorist and historian. Send in exile in Romania in 1923, he edited the periodical press « Nawasart» (1924-25) and other litterary publications in armenian and/or roumanian. Arrested in 1944 and condemned in a ten year sentence in a Siberian camp, Sirouni returns in Bucharest at the beginning of the fifties. Obsessed by the nostalgia of a country lost and haunted by the terror that came along with the years 1915-1918, Sirouni set about evoking the end of the Empire in the stories and theater pieces he wrote in the twenties and thirties. He transforms art into a modality of survival. This sort of writing is progressively transfigured in memories and historical reconstitution (Constantinople and its role, four volumes), as if the writer's mind, now fixed towards the very beginning would refuse any of the charms of fiction or autobiography dedicating itself to a more distanced examination, a more critical interpretation of the birth and the demise of the Empire. The historical narrative substitutes the literary one. We have often talked of the renunciation of literature with regard to this particular turnaround. At the same time, we can't help noticing that the Ottoman Empire revisited, in his archives, remains an objet of desire, which fascinates and tears apart at the same time the double exile that was Sirouni.

\section{INDEX}

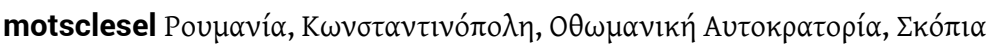

motsclesmk ОТОМАНСКАТА ИМПЕРИЈА, РОМАНИЈА, СКОПЈЕ, ЦАРИГРАД

Index chronologique : Empire ottoman

Thèmes : Littérature

motsclestr İstanbul, Romanya, Osmanlı İmparatorluğu

Mots-clés : Sirouni Hagop (1890-1973), Derviche, Sirouni Hagop (1890-1973), littérature

roumaine, littérature arménienne

Keywords : armenian diaspora, Sirouni Hagop (1890-1973), Ottoman empire, Constantinople, Literature

Index géographique : Constantinople, Roumanie 